\title{
Intermediates in formation and activity of the RNA polymerase II preinitiation complex: holoenzyme recruitment and a postrecruitment role for the TATA box and TFIIB
}

\author{
Jeffrey A. Ranish, ${ }^{1,2}$ Natalya Yudkovsky, ${ }^{1,2}$ and Steven Hahn ${ }^{2,3,4}$ \\ ${ }^{1}$ Molecular and Cellular Biology Program, University of Washington, ${ }^{2}$ The Fred Hutchinson Cancer Research Center, \\ and ${ }^{3}$ The Howard Hughes Medical Institute, Seattle, Washington 98109 USA
}

\begin{abstract}
Assembly and activity of yeast RNA polymerase II (Pol II) preinitiation complexes (PIC) was investigated with an immobilized promoter assay and extracts made from wild-type cells and from cells containing conditional mutations in components of the Pol II machinery. We describe the following findings: (1) In one step, TFIID and TFIIA assemble at the promoter independently of holoenzyme. In another step, holoenzyme is recruited to the promoter. Mutations in the CTD of Pol II, Srb2, Srb4, and Srb5, and two mutations in TFIIB disrupt recruitment of all holoenzyme components tested without affecting TFIID and TFIIA recruitment. These results indicate that the stepwise assembly pathway is blocked after TFIID/TFIIA binding. (2) Both the Gal4-AH and Gal4-VP16 activators stimulate formation of active PICs by increasing the extent of PIC formation. The Gal4-AH activator stimulated PIC formation by enhancing the binding of TFIID and TFIIA, whereas Gal4-VP16 could enhance the recruitment of TFIID, TFIIA, and holoenzyme. (3) Extracts deficient in TFIIA activity showed reduced assembly of all PIC components. These and other results suggest that TFIIA acts at an early step by enhancing the stable recruitment of TFIID. (4) An extract containing the TFIIB mutant E62G, had no defect in PIC formation, but had a severe defect in transcription. Similarly, mutation of the TATA box reduced PIC formation only two- to fourfold, but severely compromised transcription. These results demonstate an involvement of TFIIB and the TATA box in one or more steps after recruitment of factors to the promoter.
\end{abstract}

[Key Words: RNA polymerase II; holoenzyme; transcription]

Received October 16, 1998; revised version accepted November 19, 1998.

The RNA polymerase II (Pol II) transcription machinery includes six general transcription factors (GTFs; TFIIA, TFIIB, TFIID, TFIIE, TFIIF, and TFIIH), Srb and Med factors, and Pol II (Orphanides et al. 1996). The first step in transcription initiation is recruitment of the transcription machinery to the promoter to form a preinitiation complex (PIC). The efficiency of this step is determined by the accessibility of the promoter embedded in chromatin, promoter-specific regulatory factors, and the core promoter elements [TATA box, initiator (Inr), TFIIB-recognition element (BRE) and downstream promoter element (DPE)]. On the basis of order of addition experiments with purified GTFs and Pol II, a stepwise pathway for the assembly of these factors into the PIC was proposed (for review, see Orphanides et al. 1996). In this

${ }^{4}$ Corresponding author.

E-MAIL shahn@fred. fhcrc.org; www.fhcrc.org/science/basic/labs/hahn; FAX (206) 667-6497. stepwise model, TBP (a TFIID subunit) binding to the TATA box serves as a platform for the recruitment of TFIIB. Next, Pol II and TFIIF bind to the promoter, after which TFIIE and TFIIH bind. The requirement for TFIIA depends on the composition of the system. TFIIA stimulates transcription in crude systems and in systems reconstituted with pure factors when TFIID is used (for review, see Orphanides et al. 1996). When purified factors are used, TFIIA can enter the PIC at any point after TBP binding to the TATA box.

This stepwise model for PIC assembly has been challenged by the discovery of holoenzyme complexes containing Pol II, a subset of GTFs, and other factors (for review, see Chang and Jaehning 1997; Myer and Young 1998). An alternative model postulates that holoenzyme is recruited to the promoter in vivo, bypassing the need to recruit all of the factors individually. Several holoenzyme complexes in yeast and human systems have been described. One form of yeast holoenzyme contains Pol II, 
TFIIF, and the Srb/Mediator complex. This Srb/Mediator complex contains Srb2, Srb4, Srb5, Srb6, Srb7, Gal11, Rgr1, Sin4, Rox3, and 6 Med proteins (Myers et al. 1998), some of which have been implicated in Pol II transcription through genetic studies. In vitro, this holoenzyme is competent for transcription in the presence of added TBP, TFIIB, TFIIE, and TFIIH. Another form of yeast holoenzyme contains the factors noted above as well as TFIIB, TFIIH, additional Srb proteins, and SWI/SNF proteins (Wilson et al. 1996). A third form of yeast holoenzyme contains TFIIB, TFIIF, TFIIS, Gal11, Paf1, Cdc73, CCR4, and Pol II (Chang and Jaehning 1997).

Although the exact composition of the holoenzymes are variable, a number of observations support the holoenzyme recruitment model. First, essentially all of the cellular Srb proteins are found associated with $\sim 20 \%$ of cellular Pol II (Koleske and Young 1995). Second, temperature-sensitive mutations in Srb4 and Srb6 can produce a rapid shutdown in mRNA synthesis from many Pol II promoters (Thompson and Young 1995). Third, artificial recruitment of holoenzyme components to the promoter can partially bypass the need for an activator (Keaveney and Struhl 1998, and references therein). The identification of different Pol II-containing complexes suggests the possibility that distinct holoenzyme complexes may be recruited to different promoters. Whereas mutations in Srb/Mediator components can affect the expression of a broad spectrum of genes, mutations in components of the the Paf1/Cdc73 holoenzyme appear to only affect expression of a subset of genes (for review, see Chang and Jaehning 1997). Also, recent reports describe a subset of genes whose transcription is apparently independent of the Srb/Mediator holoenzyme (Lee and Lis 1998; McNeil et al. 1998).

The fact that a number of GTFs (i.e., TFIID, TFIIA, and TFIIE) are absent from the holoenzymes, leaves the mechanism of PIC assembly in vivo unresolved. A number of possibilities exist, including: (1) a concerted reaction in which the complete transcription machinery is recruited to the promoter in one step through simultaneous interactions between holoenzyme components, TFIID/TFIIA, promoter sequences and activators; (2) a stepwise reaction in which TFIID/TFIIA recruitment facilitates the association of a holoenzyme into the complex, or vice versa; or (3) independent recruitment of TFIID/TFIIA and holoenzyme.

Another unresolved question is the role of core promoter elements in PIC assembly and function. The TATA box, Inr, and DPE serve as interaction sites for components of TFIID (for review, see Orphanides et al. 1996; Hampsey 1998). The integrity of the TATA box is important for high-level transcription from many promoters in vivo and in vitro (Wobbe and Struhl 1990; Hoopes et al. 1998), and a correlation has been observed between the lifetime of TBP-TATA complexes and transcriptional activity (Hoopes et al. 1998). However, it is unlikely that the occupancy of the TATA box is the only parameter governing transcriptional activity. In the study by Hoopes et al. (1998), equivalent occupancy of two TATA boxes by TBP resulted in different levels of transcription. In another study, a TATA mutation caused a larger defect in initiation compared with PIC formation measured by a nuclease protection assay (Jacob et al. 1994). Because TBP is known to distort the DNA on interaction with the TATA box, one model that could explain these conflicting results is that the conformation of DNA in some of the TBP-mutant TATA complexes is altered, and this change decreases the activity of the final PIC. It has also been reported that the sequence of the TATA box plays a role in transcription reinitiation (Yean and Gralla 1997).

We have used a combined genetic and biochemical approach to address the role of individual subunits of the transcription machinery and the TATA box in transcription initiation. We directly assayed PIC formation and transcription activity using extracts made from either wild-type strains or strains containing conditional mutations in specific factors. This approach allowed us to observe intermediates in PIC formation and activity caused by blocking the function of individual factors. Our results demonstrate that the stepwise assembly pathway is blocked after TFIID/TFIIA recruitment, and that the TATA box and TFIIB play additional essential roles in PIC activity after assembly of the transcription complex.

\section{Results}

\section{Promoter-dependent PIC formation}

An immobilized template system was used to study PIC assembly and activity (Choy and Green 1993; Zawel et al. 1995; Sandaltzopoulos and Becker 1998). The promoter template used was the yeast HIS4 core promoter containing the TATA box and transcription start sites (Fig. 1). A single Gal4-binding site was positioned 55-bp

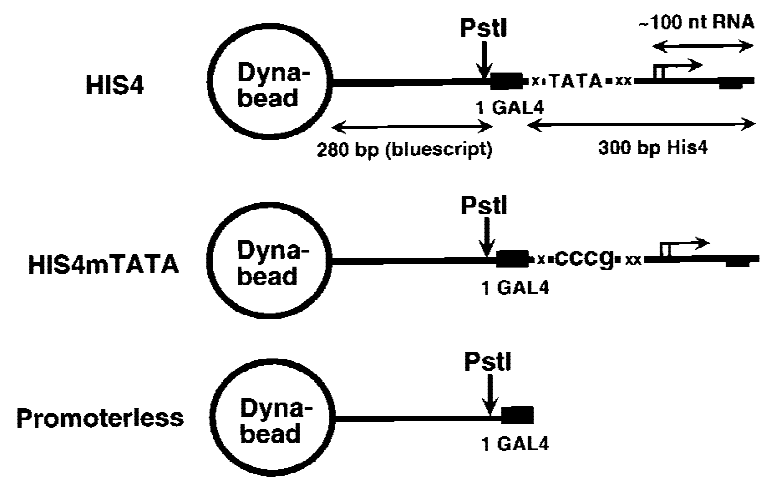

Figure 1. Immobilized templates used in this study. HIS4 consists of the HIS4 core promoter containing the TATA box and start sites of transcription, with one Gal4 site positioned upstream of the TATA box. The Pst 1 site was used for cleavage of the promoter from the beads after formation of complexes. $(\mathrm{x})$ Approximate location of bases that were changed to eliminate cryptic TATA sequences. HIS4mTATA is identical to HIS4 except the TATA box is replaced with a GC-rich sequence. Promoterless lacks the HIS4 promoter but retains the Gal4 site and upstream DNA. 
upstream of the TATA box with $280 \mathrm{bp}$ of Bluescript DNA between the Gal4 site and the upstream end of the DNA. A unique PstI site, located 20-bp upstream of the Gal4 site, was used for elution of the promoter fragments from the beads after complex formation. The template was linked to magnetic beads through a biotin-streptavidin linkage. Two other templates were used to examine the specificity of PIC formation. The first template (HIS4mTATA) is identical to the HIS4 template, but the sequence CCCGGG was substituted for the TATA box. The second template (Promoterless) contains the Gal4 site and upstream DNA, but lacks the HIS4 core promoter. The experimental design was to incubate nuclear extract with the immobilized templates for $40 \mathrm{~min}$ to allow PIC formation, wash the templates in transcription buffer to remove unbound proteins, and digest the templates with PstI to liberate the promoter with bound factors. After PstI digestion, the promoter-bound factors were separated from the beads, and these factors were analyzed by SDS-PAGE and Western blot. The activity of washed complexes was measured by resuspending the complexes in transcription buffer containing nucleoside triphosphates (NTPs) for 2-10 min. For comparison, single and multiround reactions were performed with unwashed complexes by stopping reactions 2 min or 40 60 min after addition of NTPs. RNA was isolated and analyzed by primer extension.

Figure 2a shows the results of a representative experiment in which PIC formation was measured on the HIS4 and Promoterless templates. In the absence of an activator, detectable levels of TBP, $\operatorname{TAF}_{\mathrm{II}} 90, \mathrm{TAF}_{\mathrm{II}} 30, \mathrm{TAF}_{\mathrm{II}} 20$,
Toa2 (TFIIA subunit), TFIIB, Kin28 (TFIIH subunit), and Rpb3 (Pol II subunit) were present on the HIS4 template (Fig. 2a, lane 2). In contrast, very low levels of all these factors were detected on the Promoterless template, indicating that the signals detected on HIS4 were promoter dependent (Fig. 2a, lane 8). Complexes formed on the HIS4 template were active in transcription (Fig. 2b, lanes $1,4,7)$. The pattern of $\alpha$-amanitin-sensitive RNAs produced by the washed complexes was quantitatively (within twofold) and qualitatively similar to the pattern of RNAs produced in single round transcription assays of unwashed complexes (Fig. 2b; data not shown).

To study the effect of activators on PIC assembly, the model activators, Gal4-AH and Gal4-VP16 were included in the reactions. Both activators stimulated modest increases in the levels of all factors probed for (approximately twofold for Gal4-AH and threefold for Gal4-VP16) except for TBP, which was unaffected (Fig. $2 \mathrm{a}$, cf. lanes 3 and 4 with lane 2). Although $\mathrm{TAF}_{\mathrm{II}} 90$ was not stimulated by Gal4-AH on the HIS4 template in this experiment, it was stimulated approximately twofold in three other experiments (data not shown). The stimulation of the levels of PIC components correlated well with the two- to threefold effect of the activators in washed and single round transcription assays (Fig. 2b, lanes 4-6 and 7-9). However, when Gal4-VP16 was used, significant levels of all factors were present in reactions with the Promoterless template (Fig. 2a, lane 4 vs. 10). Thus, unlike the AH activation domain, the VP16 activation domain has a strong ability to recruit PIC components independent of promoter sequences. This result is con-
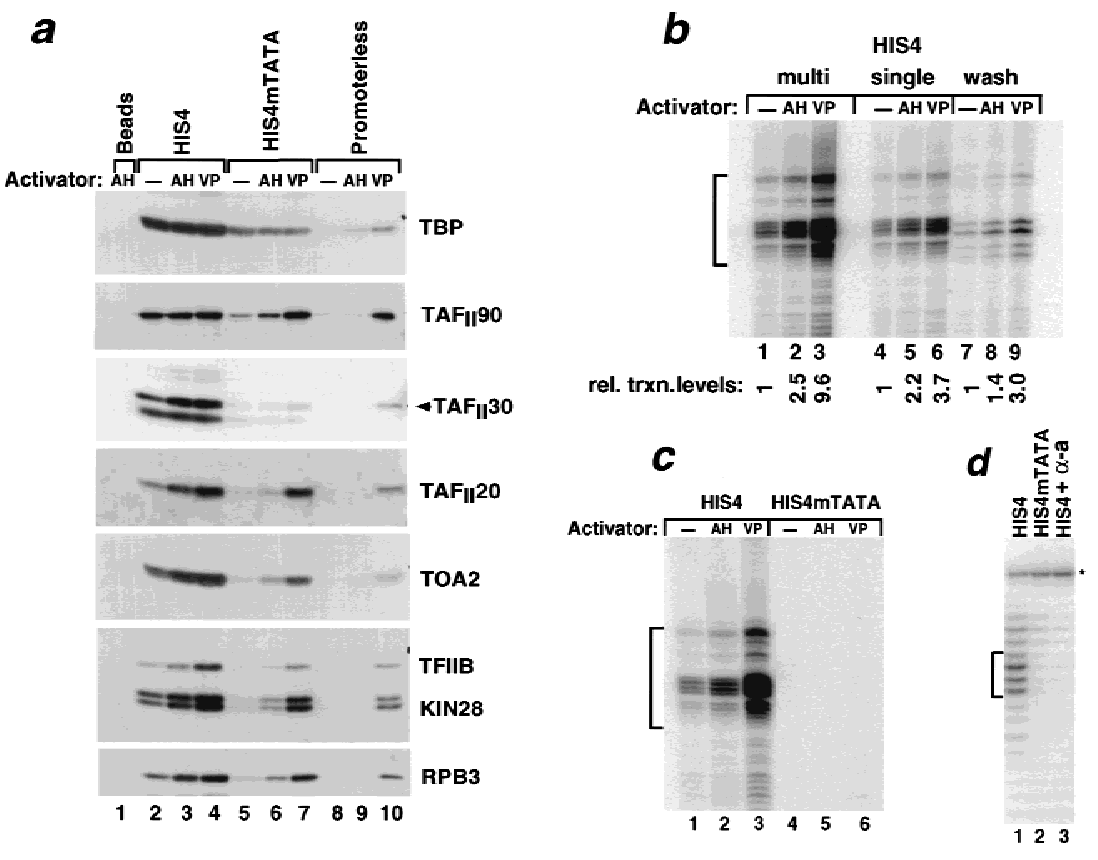

Figure 2. Promoter-dependent PIC assembly and activity. (a) PIC assembly on the HIS4, HIS4mTATA, and Promoterless templates. Nuclear extract was incubated with the indicated immobilized templates for $40 \mathrm{~min}$ with or without Gal4-AH or Gal4-VP16. PICs were isolated and analyzed as described in Methods and Materials. (Lane 1) Dynabeads lacking DNA were incubated with Gal4-AH and nuclear extract. $(b)$ Transcription activity of PICs formed on the HIS4-immobilized template. PICs were formed as described in $a$. For multiround measurements, PICs were incubated with NTPs for 60 min (lanes 1-3). Single-round measurements were obtained by incubating PICs with NTPs for 2 min (lanes 4-6). Activity of washed complexes was measured by resuspending PICs in transcription buffer containing NTPs for 10 min (lanes 7-9). Start sites were mapped by primer extension. (Brackets) $\alpha$-amanitin sensitive start sites. $(c, d)$ Transcription activity of PICs formed on the HIS4 and HIS4mTATA promoter templates. (c) PICs were formed as described in $a$ on the HIS4 (lanes 1-3) and HIS4mTATA

(lanes 4-6) immobilzed templates, and then incubated with NTPs for $40 \mathrm{~min}$. Start sites were mapped by primer extension. (d) PICs were formed on the HIS4 (lanes 1 and 3) and HIS4mTATA (lane 2) promoter templates (pSH515 and pSH514) by incubation with nuclear extract and Gal4-AH. Single-round measurements were obtained by incubating PICs with NTPs for 2 min. RNAs were detected by S1 nuclease protection assay. $\alpha$-Amanitin $(\alpha-a)$-sensitive bands used for quantitation are indicated by brackets. $\left({ }^{\star}\right)$ Position of the undigested probe. 
sistent with a report in which many PIC components were retained by a GST-VP16 column (Hengartner et al. 1995). This promoter-independent background increases with multiple Gal4 sites, and Gal4-AH can also recruit factors when three sites are present on the Promoterless template (data not shown). To avoid complications in interpreting the PIC assembly results, the remainder of the experiments were performed with Gal4-AH.

In this system, both Gal4-AH and Gal4-VP16 stimulate the levels of $\mathrm{TAF}_{\mathrm{II}} \mathrm{S}$ bound to the promoter without affecting the levels of TBP. This result indicates that a fraction of TBP can bind to the promoter without $\mathrm{TAF}_{\mathrm{II}} \mathrm{S}$ (see below). These activators also enhance PIC assembly by modestly stimulating the levels of TFIIA and holoenzyme components assembled into the PIC. This result is consistent with a number of reports suggesting that activators target TFIID, TFIIA, and/or holoenzyme components to stimulate PIC assembly (for review, see Struhl 1996).

The observation that TBP can stably bind to the promoter without $\mathrm{TAF}_{\mathrm{II}} \mathrm{S}$ is reasonable in light of findings that (1) TBP is not as tightly associated with $\operatorname{TAF}_{\mathrm{II}} \mathrm{S}$ in yeast as it is in higher eukaryotes (Reese et al. 1994), (2) a subset of $\mathrm{TAF}_{\mathrm{II}} \mathrm{S}$ are also associated with the SAGA complex (Grant et al. 1998), and (3) in yeast, TBP is in excess compared with $\mathrm{TAF}_{\mathrm{II}} \mathrm{s}$ (Lee and Young 1998). To obtain evidence that the TBP interaction was specific, we performed several controls. First, the TBP interaction site was promoter-dependent as the Promoterless template retained $<10 \%$ of the TBP retained by the HIS 4 template (Fig. 2a, lanes 2 and 3 vs. 8 and 9). Second, a comparison of PIC assembly on the HIS4 template and a template truncated $45 \mathrm{bp}$ downstream of the TATA box showed identical levels of TBP and other PIC components (data not shown). These results localized the major TBP interaction site to a 93-bp region between the Gal4 site and the DNA 45 bp downstream from the TATA box. Third, replacement of the TATA box with a SmaI site (HIS4mTATA) reproducibly resulted in at least a twofold decrease in TBP levels (with comparable decreases in all other PIC components) (Figs. 2a and 8b). It is unlikely that TBP interacts with TATA-like sequences around the TATA box as the templates were modified to eliminate these sequences /see Materials and Methods section). Taken together, these results indicate that most of the TBP signal on the HIS4 template is due to interactions with the TATA box. Because TATA-less promoters exist and a number of PIC components in addition to TBP likely interact with core promoter sequences to nucleate PIC formation (for review, see Hampsey 1998) it is possible that these interactions partially compensate for a defective TBP-TATA interaction.

\section{A special role of the TATA box}

To examine the role of the TATA box, we compared PIC formation and activity on the HIS4 and HIS4mTATA templates (Fig. 1). In basal and Gal4-AH-stimulated PIC assembly assays with HIS4mTATA, the levels of all fac- tors probed for were reduced compared with the HIS4 template (Fig. 2a, lanes 2 and 3 vs. 5 and 6, also see Fig. $8 \mathrm{~b}$, below). As mentioned above, the TBP signal was reduced at least twofold and was not stimulated by the activator. The levels of all other factors tested were reduced two- to fourfold in basal and activated reactions, but still showed an approximately twofold stimulation by Gal4-AH. As described above for the HIS4 template, complexes formed on the HIS4mTATA template were localized to the 93-bp region between the Gal4 site and the DNA 45-bp downstream of the deleted TATA box (not shown). Thus, the TATA box mutation does result in a reduction in the formation or stability of PICs. Importantly however, PIC formation is still seen in the absence of the TATA box.We next measured the transcription activity of PICs on the HIS4mTATA template. No products were detected in the primer extension assay that detects transcripts of $\sim 100$ nucleotides (Fig. 2c). To test whether these complexes were defective in synthesis of short transcripts, an S1-nuclease protection assay was used with an oligonucleotide designed to detect transcripts at least 30 nucleotides in length. In the presence of Gal4-AH, synthesis of short transcripts was reduced 13-fold by the TATA mutation (Fig. 2d). Assays to directly detect open complex formation by use of permanganate sensitivity or abortive initiation have not been successful in the yeast system (unpublished results). To test if the Pol II in these PICs was transcribing in the opposite direction, we performed primer extension reactions with a primer that recognized a sequence upstream of the Gal4 site on the nontranscribed strand. No $\alpha$-amanitin-sensitive RNAs were detected /data not shown). Thus, even though some PICs were formed on the TATA mutant template, they were severely compromised in synthesis of transcripts at least 30 nucleotides in length. These results are consistent with a role for the TATA box in the extent of PIC formation, as well as in an essential function that affects the activity of the PIC. It is possible that the destruction of the TATA sequence affects the ability of TBP to distort the DNA in a way that promotes formation of functional PICs.

\section{Use of mutant extracts to examine mechanisms of PIC formation and function}

To examine the roles of several subunits of the transcription machinery in PIC formation and function, we generated nuclear extracts from a number of strains carrying conditional alleles in specific factors. We reasoned that by using an unfractionated extract containing a mutation in a specific factor, we could ask how this defect affects PIC formation and function in the context of the remaining transcription machinery.

\section{A TBP mutant is defective in stable PIC formation}

It is well established that TBP is required for transcription of genes transcribed by Pol II as it nucleates the formation of the complete PIC (for review, see Hampsey 
1998). Using the immobilized promoter system, we assayed the ability of an extract prepared from a strain carrying the TBP I143N temperature-sensitive mutation to form active PICs. The HIS4 immobilized template was preincubated with the I143N extract and Gal4-AH, in the presence or absence of recombinant TBP (rTBP), for $40 \mathrm{~min}$, after which multi- and single-round transcription assays were performed as described for Figure $2 \mathrm{~b}$. In the absence of rTBP, very low levels of RNA were produced in multi- and single-round assays (Fig. 3a, lanes $4,6,8)$. Addition of rTBP to the mutant extract restored transcription to levels comparable with those of the wild-type extract ( 10-fold increase in single-round and washed reactions). In assays for PIC formation, the levels of all factors probed for were significantly reduced compared with the wild-type extract (Fig. 3b, lane 2). Addition of rTBP restored the levels of all PIC components tested to wild-type levels (Fig. 3b, lane 3). By densitometry, a 14-fold or greater stimulation was detected for all components except for $\mathrm{TAF}_{\mathrm{II}} 20$ and $\operatorname{TAF}_{\mathrm{II}} 90$, which showed a fourfold and twofold stimulation, respectively (Fig. 3b; data not shown). Because $\mathrm{TAF}_{\mathrm{II}} 20$ and $\mathrm{TAF}_{\mathrm{II}} 90$ were not detected on the Promoterless template in the presence of Gal4-AH, it is possible that these $\mathrm{TAF}_{\mathrm{II}} \mathrm{S}$ interact at a low level with the HIS4 promoter through a TBP-independent mechanism. Because these $\mathrm{TAF}_{\mathrm{II}} \mathrm{S}$ are also components of the SAGA complex, it is possible that their recruitment to the promoter is facilitated by its association with SAGA (Grant et al. 1998).

\section{The role of TFIIA in PIC formation and activity}

The mechanism of TFIIA action in transcription has been controversial. TFIIA has been proposed to act as both an antirepressor and a coactivator (for review, see Orphanides et al. 1996; Hampsey 1998). TFIIA interacts with numerous factors in vitro, including TBP, a TAF $\mathrm{TI}^{\prime}$ activators, and coactivators, and it can counteract the effect of a number of repressors. TFIIA can stabilize TBP binding to DNA (Weideman et al. 1997 and references therein), and it is required for an activator-dependent conformational change in TFIID-DNA complexes observed in vitro (Lieberman and Berk 1994; Chi and Carey 1996). To investigate the role of TFIIA in PIC formation and function, we prepared extracts from strains carrying conditional mutations in either of the two subunits of TFIIA, Toa1, or Toa2, for use in the immobilized template and transcription assays. Toa1-25 is a triple alanine mutation (K255, R257, K259) in the large subunit of TFIIA (Kang et al. 1995). The structure of the TFIIATBP-TATA complex and other biochemical studies suggest that the Toa1-25 mutant phenotype is due to a defective TFIIA-DNA interaction (Kang et al. 1995; Geiger et al. 1996; Tan et al. 1996). The other mutation used, Toa2-3, contains a double alanine mutation (D21 and D24) in the small subunit of TFIIA. These residues are located in the four helix bundle domain that faces away from TBP and DNA in the TFIIA-TBP-TATA complex (Geiger et al. 1996; Tan et al. 1996). In binding assays, TFIIA containing the Toa2-3 subunit formed stable complexes with TBP on DNA (Kang et al. 1995).

Extracts from strains containing the toa1-25 or toa2-3 alleles were assayed in multi- and single-round transcription experiments as described for Figure 2a. Low, but detectable, levels of transcription were detected without recombinant TFIIA (rIIA), and rIIA stimulated transcription 3- to 4-fold in single round assays (Fig. 4a, lanes 6-9 and 12-15), and 6- to12-fold in multi-round assays (Fig. 4a, lanes 4-5 and 10-11). In PIC formation assays, both mutant extracts gave reduced levels of all factors probed for, and these levels were stimulated by rIIA (Fig. 4b, lanes 2-5). The mutant TFIIAs were not detected in PICs formed in both extracts. For the Toa1-25 extract, this defect is not the result of instability of TFIIA, because both TFIIA subunits are readily detectable by Western blot (data not shown). Because our Toa2 antibodies do not react well with the Toa2-3 protein (Kang et al. 1995), we cannot quantitate the stability of this subunit in the extract. rIIA stimulated the levels of all factors tested approximately three- to fourfold [in lane 4 vs. 5, rIIA a

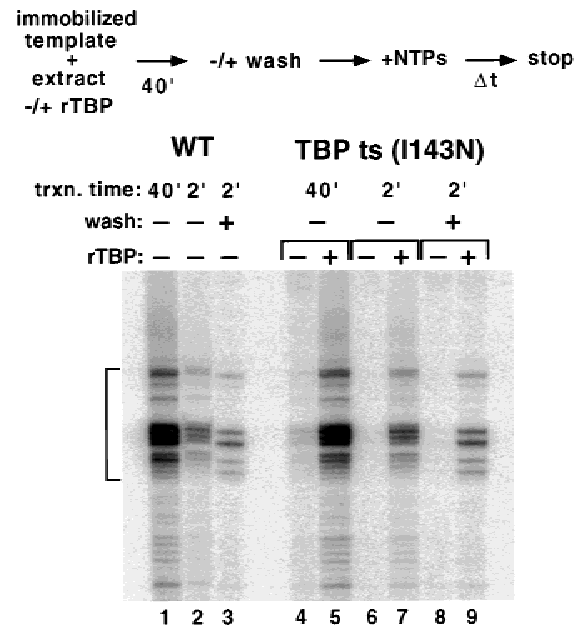

$\boldsymbol{b}$

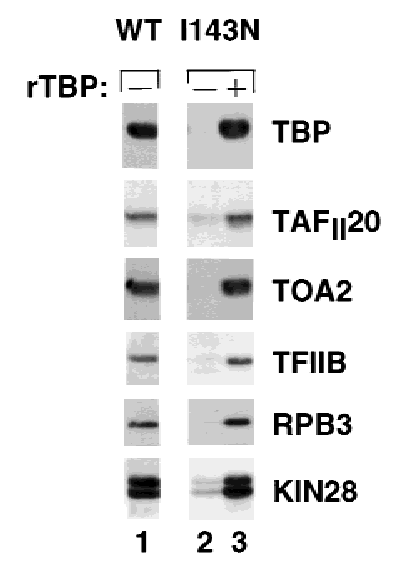

Figure 3. An extract containing a TBP mutant is defective in stable PIC formation and activity. $(a)$ Transcription activity of PICs formed in wildtype and TBP(I143N) extracts. Experimental design is outlined at top. The indicated extracts were incubated with the HIS4 immobilized template and Gal4-AH for 40 min. rTBP (200 ng) was included where indicated. For multiround measurements, PICs were incubated with NTPs for $40 \mathrm{~min}$ (lanes 1,4,5). Single round measurements were obtained by incubating PICs with NTPs for 2 min (lanes 2,6,7). Activity of washed complexes was measured by resuspending PICs in transcription buffer containing NTPs for 2 min (lanes $3,8,9)$. (Brackets) $\alpha$-Amanitin sensitive start sites. (b) PIC assembly on the HIS4 template in wildtype and TBP(I143N) extracts. PICs were formed as described in $a$ except that reactions were scaled up twofold. PICs were analyzed as described in Fig. 2a. 
Figure 4. Extracts containing mutant subunits of TFIIA are defective in stable PIC formation and activity. (a) Transcription activity of PICs formed in wild-type and TFIIA mutant extracts. Experimental design is outlined at the top. The indicated extracts were incubated with the HIS4 immobilized template and Gal4-AH for 40 min. rTFIIA (80 ng) was included where indicated. For multiround measurements, PICs were incubated with NTPs for $40 \mathrm{~min}$ (lanes 1,4,5,10,11). Single round measurements were obtained by incubating PICs with NTPs for 2 min (lanes 2,6,7,12,13). Activity of washed complexes was measured by resuspending PICs in transcription buffer containing NTPs for 2 min (lanes $3,8,9,14,15)$. Brackets indicate the $\alpha$-amanitin sensitive start sites. (b) PIC assembly on the HIS4 template in wild-type and TFIIA mutant extracts. PICs were formed as described in $a$ except that reactions were scaled up twofold. PICs were analyzed as described in Fig. 2a. a

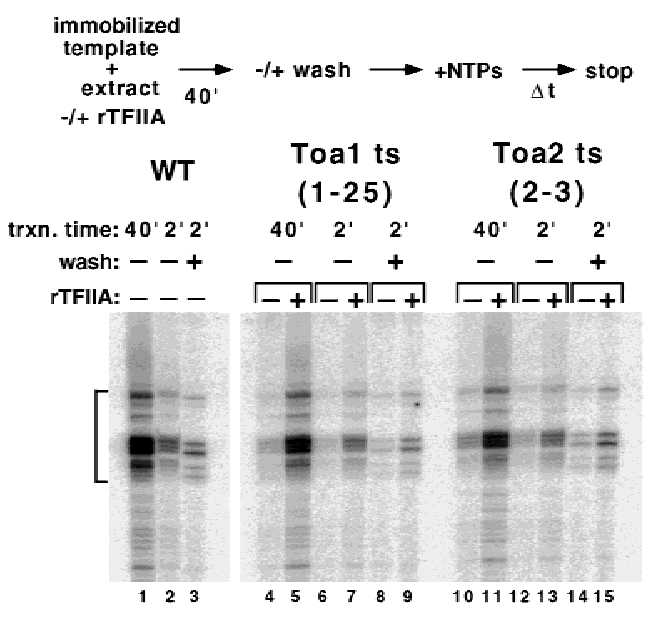

$\boldsymbol{b}$

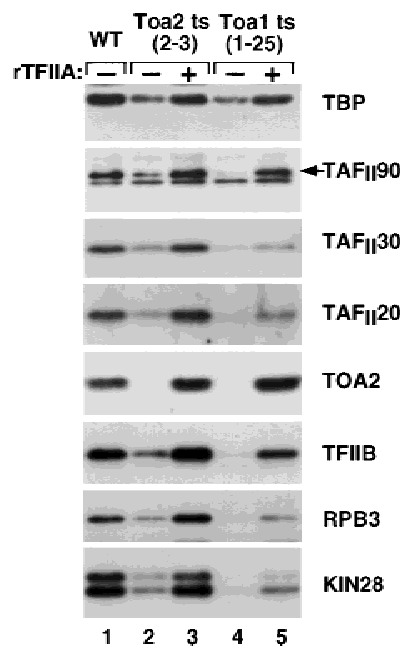

stimulates $\mathrm{TAF}_{\mathrm{II}} 90$ approximately eightfold. However, in two other experiments $\mathrm{TAF}_{\mathrm{II}} 90$ was stimulated approximately threefold (not shown)]. The correlation between the effect of TFIIA on both the activity of PICs and the levels of PIC components, suggests that TFIIA stimulates transcription by increasing the extent of PIC formation. Because we did not detect changes in the levels of NC2 $\alpha$ (Gadbois et al. 1997) in PIC formation assays in the presence or absence of TFIIA (not shown), it seems unlikely that TFIIA stimulates transcription by removal of this regulator from the templates. Our results that TFIIA affects the stable recruitment of TFIID in nuclear extracts are consistent with a number of studies using purified factors that demonstrated a role for TFIIA in the extent of formation of stable activator-TFIID-TFIIATATA complexes (Lieberman and Berk 1994; Chi et al. 1995; Kobayashi et al. 1998). An alternative model in which the defect in TFIID recruitment is a secondary effect due to a role of TFIIA in holoenzyme recruitment, is not consistent with experiments presented in the next section.

The effect of TFIIA on the extent of PIC formation could be explained by an effect on the rate of complex assembly, and/or the stability of PICs. To probe the mechanism of TFIIA action, we measured the rate of active PIC formation in the presence and absence of rIIA. In the experiment shown in Figure 5a, the Toa1-25 extract was incubated with the HIS4 template to which Gal4-AH was prebound, in the presence or absence of rIIA. At the indicated times, an aliquot of the reaction was removed to a tube containing NTPs to initiate transcription. To obtain single round measurements of RNA production, reactions were stopped after $2 \mathrm{~min}$. The results show that during the $\sim 40$ min window during which active PICs accumulate, TFIIA stimulates the rate of active PIC formation. Measurements of the slopes of the curves in Figure 5b show that TFIIA has at least a fivefold effect on the rate of active PIC formation. We next tested whether the stimulation of PIC formation by TFIIA could be explained by an increase in stability of PICs. To do this, we used the template challenge protocol outlined in Figure 5c. PICs were allowed to form for $40 \mathrm{~min}$ in the presence or absence of rIIA, after which a 20-fold molar excess of challenger HIS4 template was added for up to $60 \mathrm{~min}$. The number of active PICs that were stable to challenge was measured by adding NTPs to the reactions for $2 \mathrm{~min}$, followed by stop buffer. Addition of the challenge template at the start of the reaction strongly inhibited transcription, showing that the challenge template could effectively compete for transcription factors in these reactions (Fig. 5c, lanes 1 vs. 2 and 6 vs. 7). However, after $60 \mathrm{~min}$ of challenge, PICs formed in the absence and presence of rIIA were relatively stable (Fig. 5c, lanes 4 vs. 5 and 9 vs. 10). By PhosphorImager analysis, $\sim 50 \%$ and $55 \%$ of the the active PICs remained stable to challenge in the absence and presence of rIIA, respectively. Together with previous results, these findings suggest that TFIIA stimulates transcription by promoting the stable association of TFIID with the promoter. As a result, the rate and extent of PIC formation is increased. However, once formed, PICs that lack TFIIA are stable and active to the same extent as complexes containing TFIIA.

\section{Mutations in TFIIB, Pol II, and Srbs affect a step in PIC assembly distinct from TFIID / TFIIA recruitment}

To gain a more complete understanding of steps in PIC formation and function, we prepared nuclear extracts from strains carrying mutant alleles of Sua7 (TFIIB), Rpb1 (the large subunit of Pol II), and Srb2, Srb4, and 


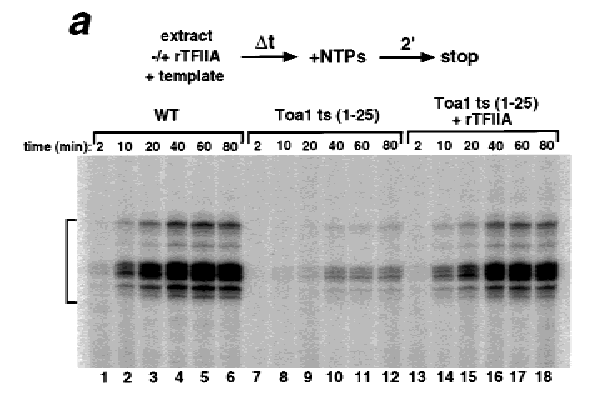

b

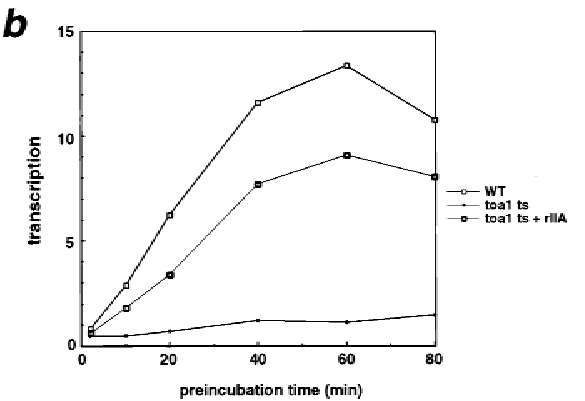

RNA Pol II preinitiation complex intermediates

Figure 5. Mechanism of TFIIA action. (a) Rate of formation of active PICs in a wildtype and a Toa 1-25 mutant extract. Experimental design is outlined at top. The indicated nuclear extracts were incubated with the HIS4 template (pSH515) and Gal4-AH, in the presence or absence of rTFIIA (40 ng). At the indicated time, an aliquot of the reaction was removed and added to a tube containing NTPs. After 2 min the reaction was stopped and analyzed by primer extension. (Brackets) $\alpha$-Amanitin- sensitive start sites. $(b)$ Graph of the quantitated data from $a$. $(c)$ Stability of PICs to challenge by a second template in the presence and absence of TFIIA. Experimental design is outlined at top. Toal-25 mutant extract was incubated with the HIS4 template (pSH515) and Gal4-AH for 40 min. rTFIIA (40 ng) was included in reactions $6-10$. PICs were challenged by addition of a 20 -fold molar excess of a HIS4 promoter template (pSH388) for either 10 min (lanes 3,8) or 60 min (lanes 4,9). Transcripts produced from this template are not detected with the primer used in these experiments. Single-round transcription was measured by addition of NTPs for $2 \mathrm{~min}$. (Lanes 1,6) Single-round transcription of PICs formed for 40 min with no challenge, in the absence and presence of rTFIIA. (Lanes 2,7) Identical to lanes 1 and 6 except that pSH388 was added at the start of the incubations. (Lanes 5,10) Single-round transcription of PICs that have incubated for the same time as reactions 4 and 9 without pSH388.

Srb5, and assayed them for transcription and PIC formation. This combined genetic and biochemical approach allowed us to test models of PIC formation. The holoenzyme model predicts that disruption of holoenzyme would block the assembly of all holoenzyme components. In contrast, the stepwise model predicts that factor disruption would not impair assembly of any factors preceding the affected step.

We first examined the role of TFIIB in stable PIC assembly by preparing nuclear extracts from three strains carrying conditional alleles in Sua7 for use in the immobilized template assay. The strains used in this study have missense mutations in the zinc ribbon domain (G41E), the conserved amino-terminal region, carboxyterminal to the zinc ribbon (E62G), and the first repeat region (C149R) (Knaus et al. 1996). Extracts prepared from these strains were tested for the ability to form active PICs by single round transcription assays (see Materials and Methods). The Sua7(G41E) extract gave the lowest levels of transcription, but levels were stimulated

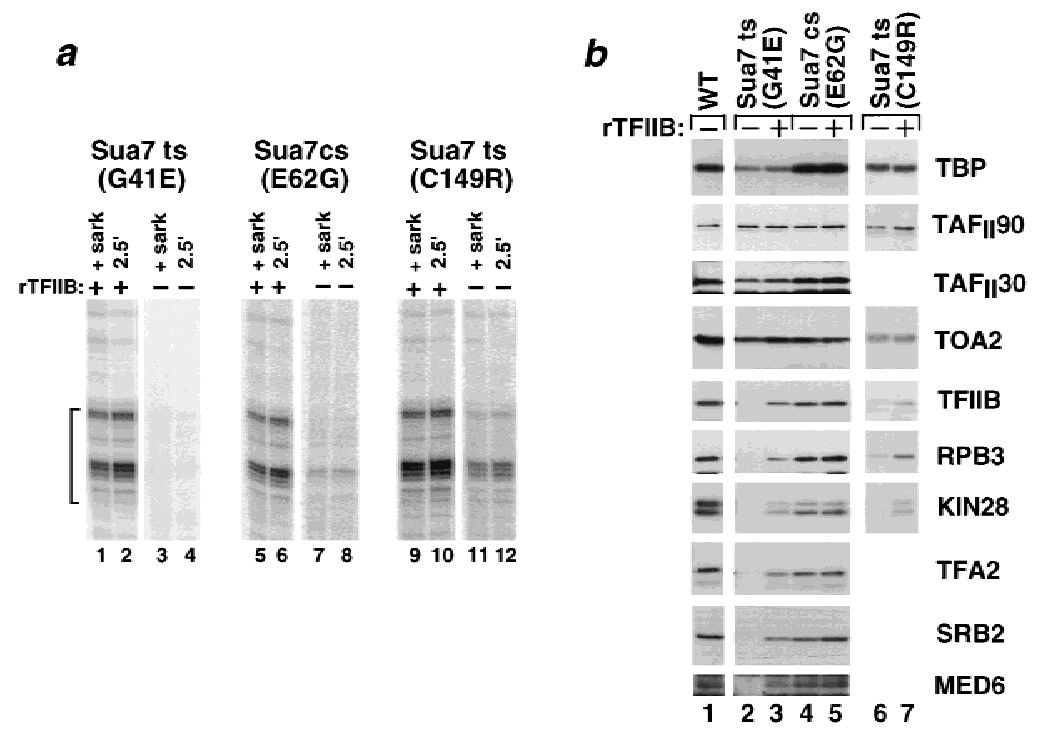

Figure 6. Analysis of TFIIB mutant extract in PIC formation and activity. (a) Transcription activity of PICs formed in TFIIB mutant extracts. The indicated extracts were incubated with the HIS4 promoter template (pSH515) and Gal4-AH for 35 min. rTFIIB (30 ng) was included where indicated. After addition of NTPs, single-round transcription was measured by two methods: (1) sarkosyl was added to $0.2 \%$ after $45 \mathrm{sec}($ lanes 1,3 ) or 1 minute (lanes $5,7,9,11)$, followed by SDS stop buffer after $10 \mathrm{~min}$; or (2) SDS stop buffer was added after $2.5 \mathrm{~min}$ (lanes 2,4,6,8,10,12). (Brackets) $\alpha$-Amanitin-sensitive start sites. (b) PIC formation in TFIIB mutant extracts. PICs were formed by incubation of the indicated extracts with the HIS4 immobilized template and Gal4-AH for 40 min. rTFIIB (120 ng) was included in the incubation where indicated. PICs were analyzed as described in Fig. 2a. 
20-fold by rIIB (Fig. 6a, lanes 1-4). Sua7(E62G) and Sua7(C149R) extracts gave low levels of transcription that were stimulated 4- to 5-fold [Sua7(E62G)] and 2.8fold [Sua7(C149R)] by rIIB (Fig. 6a, lanes 5-8 and 9-12). To test if these effects were attributable to defects in PIC formation, immobilized template assays were performed. Whereas all TFIIB mutant extracts showed no stimulation of TFIID or TFIIA by rIIB, the Sua7(G41E) and Sua7(C149R) extracts showed reduced levels of all other components probed for (Fig. 6b, lanes 2 vs. 3 and 6 vs. 7). For Sua7(G41E), Tfa2 (small subunit of TFIIE) and holoenzyme components, including TFIIB, Rpb3, Kin28, Med6, and Srb2, were stimulated at least fourfold by rIIB. For Sua7(C149R), holoenzyme components were stimulated at least twofold by rIIB. In general, these extracts show a good correlation between the stimulation of transcription and levels of holoenzyme components by rIIB. These results are consistent with models in which TFIIB is recruited to the PIC in a step that is distinct from TFIID/IIA recruitment (with or without holoenzyme components), and functional TFIIB is required for the stable assembly of the rest of the PIC components. In contrast, in PIC formation assays with the Sua7(E62G) extract, the levels of all factors probed for were unaffected by rIIB. Because the number of active PICs was stimulated approximately fourfold by rIIB without any effect on the extent of PIC formation, this mutant defines a role for TFIIB in the activity of PICs that is distinct from its requirement in stable PIC assembly.

To test the role of the CTD of Pol II in PIC assembly and function, we prepared an extract from a strain carrying a conditional allele of $R p b 1$ that lacks all but 9 of the 26 heptapeptide repeats of the CTD of the large subunit of Pol II (West and Corden 1995). The integrity of the CTD is required for normal levels of activated transcription at a number of promoters in vivo and in vitro (Liao et al. 1991 and references therein; Gerber et al. 1995). Because the Srb/Mediator complex interacts with Pol II through the CTD, genetically and biochemically, it is likely that this mutation affects the integrity of this holoenzyme complex (for review, see Koleske and Young 1995). In single- and multi-round transcription assays, extracts prepared from this strain were severely impaired for transcription (Fig. 7a). Compared with an extract from a wild-type strain, the CTD9 extract shows at least a 25-fold reduction in single-round transcription in the presence of Gal4-AH (Fig. 7a, lanes 2 and 3 vs. 5 and 6). To test whether this transcription defect was the result of a failure to form stable PICs, we used this extract in the immobilized template assay. Interestingly, compared with the TFIID and TFIIA components, very low levels of holoenzyme components (TFIIB, Kin28, and Rpb3) were detected in reactions with the CTD9 extract. Compared with the wild-type extract, TFIID and TFIIA levels were nearly normal, whereas the TFIIB and Kin28 levels were reduced $>10$-fold in the CTD9 extract. These results suggest that (1) the CTD9 transcription defect is due to a defect in stable recruitment of holoenzyme components, and (2) TFIIB is recruited via a holoenzyme complex, rather than in a stepwise fashion, in our system.

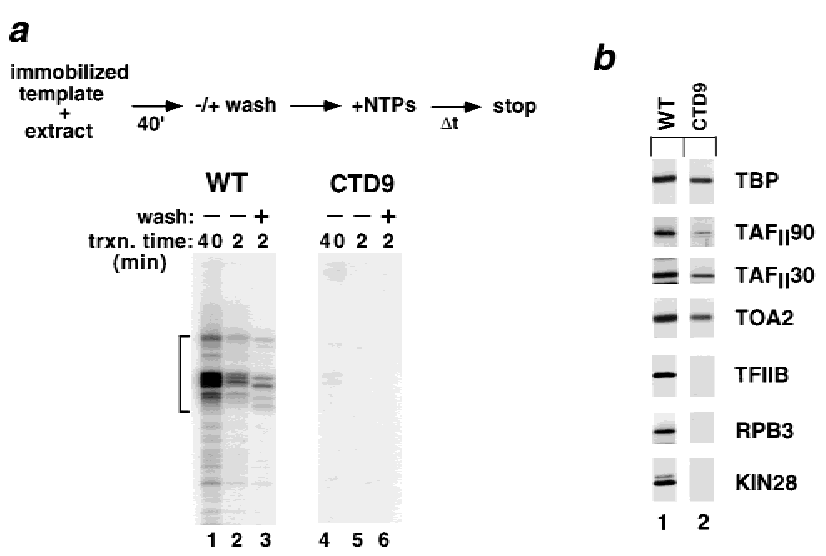

Figure 7. The role of the CTD of Pol II in PIC formation and activity. (a) Transcription activity of PICs formed in a wild-type and a CTD9 extract. Experimental design is outlined at top. The indicated extracts were incubated with the HIS4 immobilized template and Gal4-AH for $40 \mathrm{~min}$. For multi-round measurements, PICs were incubated with NTPs for 40 min (lanes 1,4). Single-round measurements were obtained by incubating PICs with NTPs for 2 min (lanes 2,5). Activity of washed complexes was measured by resuspending PICs in transcription buffer containing NTPs for 2 min (lanes 3,6). (Brackets) $\alpha$-Amanitin-sensitive start sites. (b) PIC assembly on the HIS4 immobilized template in wild-type and CTD9 extracts. PICs were formed as described in $a$ except that reactions were scaled up twofold. PICs were analyzed as described in Fig. 2a.

As a further test of the holoenzyme recruitment models, we tested PIC assembly and function in extracts containing mutations in Srb proteins. The Srbs were originally identified as suppressors of the cold-sensitive phenotype caused by CTD truncation (for review, see Koleske and Young 1995). Transcription assays were performed with nuclear extracts from yeast strains that contained deletions in either Srb2 $(\Delta \mathrm{Srb} 2)$ or Srb5 $(\Delta \mathrm{Srb} 5)$, or contained a temperature-sensitive mutation in Srb4 (Srb4ts). All of the extracts were defective in both single and multi-round transcription (Fig. 8a). The $\Delta$ Srb2 and $\Delta$ Srb5 extracts were the most severely defective, with a $>20$-fold decrease in single-round transcription as compared with the wild-type extract (Fig. 8a, lanes 1 and 2 vs. $4,5,10$, and 11). Addition of recombinant Srb2 (rSrb2) to the $\Delta \mathrm{Srb} 2$ extract restored transcription to levels that were at least $50 \%$ of wild-type (Fig. $8 \mathrm{a}$, lanes 1 and 2 vs. 7 and 8). The Srb4ts extract showed an approximately sixfold reduction in single-round transcription as compared with the wild-type extract (Fig. 8a, lanes 1 and 2 vs. 13 and 14). We then tested whether these defects in transcription could be due to a failure to form stable PICs. All of the extracts showed essentially wild-type levels of TBP, TAF $_{\text {II }} 90$, and Toa2, but reduced levels of all the holoenzyme components probed for (Fig. 8, b, lanes 1,3, and 5 , and $8 \mathrm{c})$. Specifically, the $\Delta \mathrm{Srb} 2$ and $\Delta$ Srb5 extracts showed decreases of $>16$-fold in levels of Pol II, TFIIB, Kin28, Srb2, and Srb4. The Srb4ts extract showed decreases of between 5 - and 20-fold in these same holoenzyme components. Because the Srb4 signal is reduced in 
a

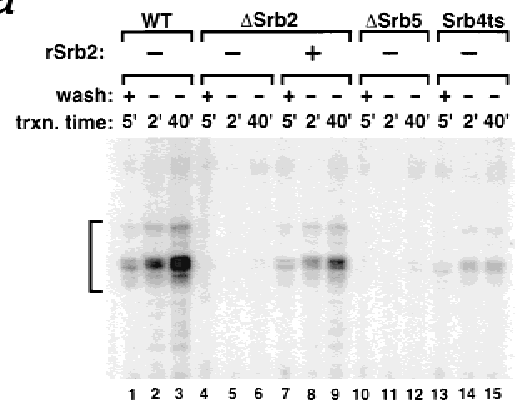

b
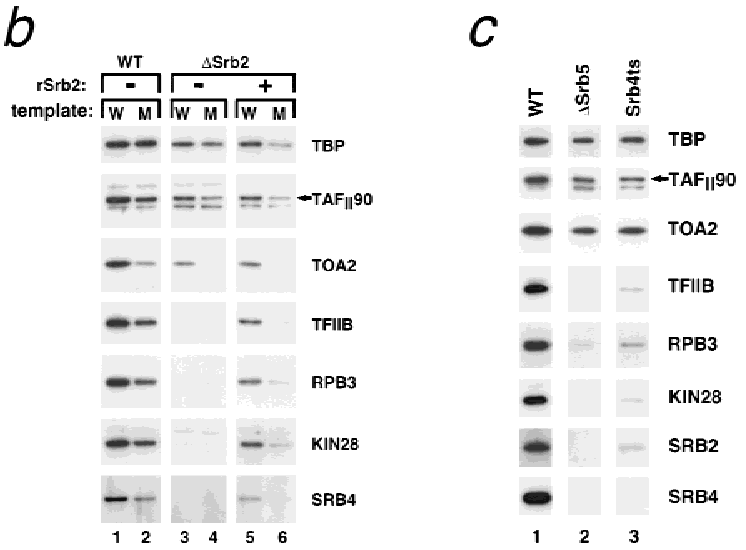

Figure 8. The role of Srb2, Srb4, and Srb5 in active PIC formation. (a) Transcription activity of PICs formed in a wild-type extract, and in Srb2, Srb4, and Srb5 mutant extracts. The indicated extracts were incubated with the HIS4- immobilized template and Gal4-AH for $40 \mathrm{~min}$. rSrb2 (100 ng) was added to reactions 7-9. For multiround measurements, PICs were incubated with NTPs for 40 min (lanes 3,6,9,12,15). Single-round measurements were obtained by incubating PICs with NTPs for 2 min (lanes 2,5,8,11, and 14). Activity of washed complexes was measured by resuspending PICs in transcription buffer containing NTPs for 5 min (lanes 1,4,7,10,13). (Brackets) $\alpha$-Amanitin-sensitive start sites. (b) PIC assembly on the HIS4 (W) and HIS4mTATA (M) immobilized templates in a wild-type extract and a Srb2 mutant extract. PICs were formed as described in $a$ except that reactions were scaled up twofold. rSrb2 was included in reactions 5 and 6 . PICs were analyzed as described in Fig. 2a. (c) PIC assembly on the HIS4-immobilized template in a wild-type extract and in Srb4 and 5 mutant extracts. PICs were formed as described in $a$ except that reactions were scaled up twofold. PICs were analyzed as described in Fig. 2a.

the Srb4ts extract, its absence in lane 3 may be the result of instability or reduced immune reactivity (not shown). Addition of rSrb2 did not affect levels of bound TBP, $\operatorname{TAF}_{\text {II }} 90$, and Toa2, but did restore the stable assembly of holoenzyme components into PICs (Fig. 8b, lane 3 vs. 5). The relative levels of PICs formed corresponded well with the relative levels of transcription seen with each extract. In addition, the levels of all PICs were enhanced by the presence of a functional TATA box (Fig. 8b, lanes $1,3,5$ vs. $2,4,6$, and data not shown). These results are very similar to those seen above with the CTD9, Sua7(G41E), and Sua7(C149R) extracts. Taken together, our results indicate that: (1) mutations in the CTD,
TFIIB, Srb2, Srb4, or Srb5 can disrupt the ability of all holoenzyme components to stably assemble at the promoter, (2) the stepwise assembly pathway is blocked after TFIID/TFIIA binding, and (3) an Srb/Mediator form of holoenzyme is recruited to the promoter in this study. In support of the latter point, Srb2, Srb4, Med6, and Swi3 were detected in PICs (Figs. 6b, and 8b,c; data not shown). However, CCR4, a component of the Cdc73/ Paf1 holoenzyme (Chang and Jaehning 1997) was absent from PICs (data not shown).

\section{Discussion}

We have used an immobilized promoter assay to study events leading to formation of active PICs. This assay, in combination with mutations in various subunits of the transcription machinery allowed us to examine the role of selected factors and the TATA box in transcription initiation. Several facts suggest that the immobilized template assay detects authentic PICs assembled at the promoter. First, binding of the transcription machinery is strongly dependent on a promoter. Second, PIC formation is TBP dependent. Third, an excellent correlation was observed between the relative amounts of factors in the PICs and the relative levels of single-round transcription observed with or without activators. Fourth, mutations in TBP, TFIIA, Pol II, Srb2, Srb4, Srb5, and two mutations in TFIIB reduce PIC formation by an amount that corresponds closely to the reduction seen in singleround transcription. Fifth, analysis of PICs by quantitative Western blots revealed that TFIIA, TFIIE, and TFIIB were present in stoichiometric amounts /data not shown). A model summarizing our results is presented in Figure 9.

We have measured the amount of RNA produced from yeast PICs and found that only a fraction of the complexes are active in productive initiation (J.A. Ranish and S. Hahn, unpubl.). However, it seems that most of the mutations studied here do not affect the fraction of PICs that are active because there is an excellent correlation between the relative transcription activity and the relative amounts of PICs observed with wild-type and mutant extracts. Thus far, the only mutations we have found that decrease the activity of the PICs are the TATA mutation and the Sua7(E62G) mutation.

\section{Extracts from conditional yeast strains define at least two intermediates in PIC assembly}

PIC formation requires assembly of $>50$ polypeptides at the promoter. The identification of preassembled holoenzyme complexes containing Pol II and a subset of the transcription machinery suggested that at least some PIC assembly involves the recruitment of preassembled factors. However, a number of important questions about the assembly of the PIC remain unanswered. First, not all factors are in the holoenzyme complexes and it is unclear how the preassembled factors interact with the other factors to form the final PIC. Second, at least three forms of yeast holoenzymes have been isolated and it is 


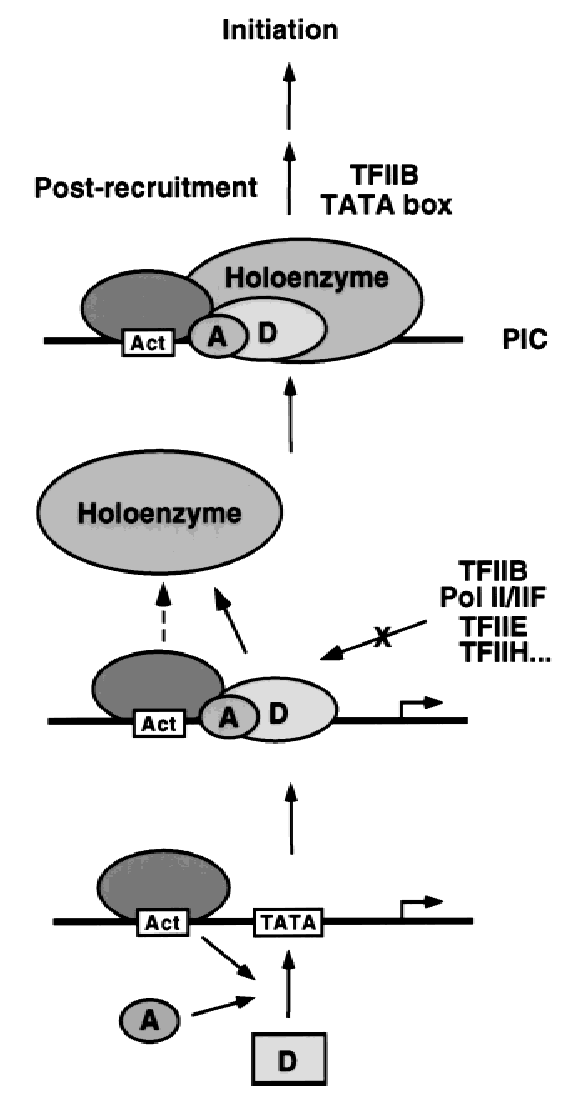

Figure 9. Mechanism of PIC assembly. In the model, TFIID and TFIIA are stably recruited to the promoter by an activator. In the presence of Gal4-AH, the D-A-promoter complex is required for the stable recruitment of the Srb/Mediator holoenzyme. The strong activator Gal4-VP16 can recruit holoenzyme independently of the D-A-promoter complex. Importantly, after D-A recruitment, the stepwise assembly pathway is blocked. Alternative PIC assembly mechanisms are possible, including a concerted reaction or holoenzyme independent PIC formation depending on the promoter and activators involved. A postrecruitment role for the TATA box and TFIIB, such as open complex formation or promoter clearance, is indicated.

not clear which form assembles at any particular promoter. Third, there is an excess of free general factors over holoenzyme complexes (Lee and Young 1998), suggesting that stepwise PIC assembly is also a viable mechanism.

We found that extracts containing mutations in TBP or TFIIA subunits showed defects in stable recruitment of all factors tested with the GAL4-AH activator. Under these conditions, holoenzyme was not recruited independently of TFIID and TFIIA. In contrast, extracts containing mutations in TFIIB, Pol II, Srb2, Srb4, and Srb5 showed defects in recruitment of all components except TFIID and TFIIA. These results indicate that in the yeast system, PIC assembly can occur in at least two steps: the holoenzyme-independent assembly of TFIID and TFIIA at the promoter, followed by holoenzyme recruitment. However, it is likely that alternative mechanisms exist depending on promoter architecture, the types of regula- tory factors present, and the presence of chromatin (Struhl 1996). For example, holoenzyme recruitment could precede TFIID recruitment. The Gal4-VP16 activator can recruit high levels of holoenzyme components (Hengartner et al. 1995) and certain $\mathrm{TAF}_{\mathrm{II}} \mathrm{s}$ in a manner that does not require promoter DNA (Fig. 2a). In vivo, artificial recruitment of holoenzyme components can bypass the need for an activator (Keaveney and Struhl 1998 and references therein). One interpretation of these experiments is that activators such as Gal4-VP16 target holoenzyme components as well as TFIID for recruitment. Also, alternative mechanisms for PIC assembly likely exist at promoters that are transcribed independently of Srb factors (Lee and Lis 1998; McNeil et al. 1998).

As it has been estimated that TFIIB and the other general transcription factors are in 5- to 10-fold excess over the holoenzyme complex (Lee and Young 1998), it is surprising that little detectable binding of TFIIB to the TFIID-TFIIA-promoter complex occurs in the Pol II or Srb mutant extracts (Figs. $7 \mathrm{~b}$ and $8 \mathrm{~b}, \mathrm{c}$ ). Western blot analysis showed that TFIIB is present at normal levels in these extracts. One possibility is that a repressor or some modification blocks binding of TFIIB to the TFIIDTFIIA-promoter complex when it is not part of a holoenzyme complex. One important unanswered question is why Srbs are required for transcription in crude yeast extracts (for review, see Koleske and Young 1995), but not required in highly purified systems. On the basis of our results, one possible reason for the Srb requirement in crude extracts is that the stepwise assembly pathway is blocked. Interestingly, it was found that mutation of two transcription repressors (NC2 or the NOT complex) could partially compensate for a temperature-sensitive mutation in Srb4 (Gadbois et al. 1997; Lee et al. 1998). Further work will be required to explore the relationship between these and other repressors and the requirement for Srb factors in transcription initiation. However, initial experiments have shown that a mutation in $\mathrm{NC} 2 \alpha$ (Gadbois et al. 1997; Lee et al. 1998) will not compensate for a mutation in Srb4 in vitro (S. Hahn, unpubl.).

\section{The role of TFIIA in PIC assembly and activity}

In systems that are responsive to TFIIA, TFIIA stimulates both basal and activated transcription (DeJong et al. 1995; Kang et al. 1995). TFIIA tightly binds TBP on DNA and this binding competes with inhibitors of TBP-DNA binding, such as yTAF ${ }_{\text {II }} 145 / \mathrm{hTAF}_{\mathrm{II}} 250$ and Mot1 (Auble and Hahn 1993; Kokubo et al. 1998; Ozer et al. 1998). TFIIA binding to TBP also competes with other repressors that bind TBP and inhibit the assembly of active PICs such as, NC2, topoisomeraseI/Dr2, and HMG1 (for review, see Hampsey 1998). These results have led to the model that TFIIA functions to counteract inhibitors of transcription by blocking access to TBP. Several in vivo studies suggest that TFIIA may play a specific role in promoting high levels of activated transcription distinct from its mechanism of counteracting transcription inhibitors. In these studies, yeast and human TBP muta- 
tions were found that specifically inhibited activated transcription, and these mutations were defective in TFIIA binding (Stargell and Struhl 1995; Bryant et al. 1996). In vitro, TFIIA stimulates the rate and extent of purified TFIID binding to DNA, and activators can enhance this effect (Chi et al. 1995; Kobayashi et al. 1998). Additionally, it was found that the form of TFIID that binds in the presence of an activator and TFIIA is in a different conformation than that which weakly binds in the absence of TFIIA and/or activator (Lieberman and Berk 1994; Chi and Carey 1996). Furthermore, TFIIA has been found to interact directly with certain activators and coactivators in vitro, and it has been proposed that these protein-protein interactions are important for specific stimulation of activated levels of transcription (for review, see Hampsey 1998).

With extracts from strains carrying conditional mutations in TFIIA subunits, PIC formation was defective. The levels of all components examined were reduced, including TBP and $\mathrm{TAF}_{\mathrm{II}} \mathrm{s}$. It is unlikely that the defect in TFIID recruitment is a secondary effect due to a role of TFIIA in holoenzyme recruitment, because TFIID recruitment is unaffected in extracts from strains containing mutations in holoenzyme components. The defects in PIC formation caused by the TFIIA mutations correlated well with defects in the transcription activity of these PICs. TFIIA stimulated the rate, as well as the extent, of active PIC formation, but complexes that lacked TFIIA were just as stable as complexes that contained TFIIA. Our results, using a crude system, together with previous results using purified factors, demonstrate that TFIIA acts by stimulating the rate and extent of TFIID binding to the promoter.

Taken together with previous findings, our results lead to the following model for TFIIA action: TFIID exists in two forms, a productive form that is capable of stably interacting with the promoter, and a nonproductive form that cannot stably bind the promoter because of the inhibitory effect of $\mathrm{TAF}_{\mathrm{II}} \mathrm{S}$, other repressors, and dimerization. TFIIA shifts this equilibrium toward the productive form of TFIID resulting in more stable recruitment of TFIID. By this model, the productive form of TFIID that binds DNA would be analogous to the form observed in the presence of activator, TFIID, and TFIIA with purified factors (Lieberman and Berk 1994; Chi and Carey 1996). This model can account for TFIIA's requirement for activated transcription because high levels of activatorTFIID-TFIIA complexes will be stably bound to the promoter in which they can support multiple rounds of transcription. It is unlikely that the only role of TFIIA is to counteract the effect of Mot1, because in extracts prepared from a TFIIA/Mot1 double-mutant strain, TFIIA still stimulated transcription (data not shown). Also, it is unlikely that TFIIA acts solely by removal of the repressor NC2 from the promoter, because the levels of this factor at the promoter were unaffected by the TFIIA mutations (data not shown). However, it is possible that TFIIA counteracts NC2 binding to TBP off of DNA, which could increase the pool of TFIID that can stably interact with the promoter.

\section{A postrecruitment function for TFIIB}

One extract containing the TFIIB mutation E62G has a strong transcription defect, but is unaffected for PIC assembly. These results suggest that TFIIB has a postrecruitment role that is independent of its role in PIC assembly. Residue E62 is in the amino-terminal conserved region of TFIIB, and it lies within a homology block (residues 61-68) that is conserved among all the TFIIB family members (Pardee et al. 1998). Another mutation of this same residue, $\mathrm{E} 62 \mathrm{~K}$, results in a downstream shift in start site selection and a defect in Pol II interaction (Pinto et al. 1994; Bushnell et al. 1996). Other mutations in this homology block alter start site selection without affecting Pol II interaction (Pardee et al. 1998). Although we did not detect altered start site selection, our results with the E62G mutant are consistent with the latter results because, in both cases, a step after PIC recruitment was affected. Our data suggest that PICs assembled with the E62G mutant are defective at a step after PIC formation such as open-complex formation or promoter clearance.

Recently, a large deletion in the Pol III factor Brf (TFIIB related factor), was described that recruits Pol III normally, but results in a defect in open-complex formation (Kassavetis et al. 1998). This deletion removes the homology block described above in TFIIB as well as the zinc ribbon and the first repeat in the TFIIB-related region. It is possible that the defect in this Brf deletion and the E62G TFIIB mutant result in analogous defects in initiation for both Pol II and Pol III.

\section{A special role for the TATA box}

The role of the TATA box as an important promoter element and as a target for TBP binding is firmly established. Most, but not all, TATA mutations that decrease transcription have a corresponding effect on TBP binding (Hoopes et al. 1998). However, few studies on TATA box function have directly monitored the effects of TATA mutations on PIC formation. We found that removal of the TATA box severely reduced single- and multi-round transcription. Surprisingly, PIC assembly was decreased by only two- to fourfold, in contrast to the dramatic decrease in transcription activity. It is likely that other elements present at the promoter as well as protein-protein interactions, compensate for the TATA box mutation to permit recruitment of factors to form a stable PIC. Our results are consistent with a previous study in which a nuclease protection assay was used to monitor PIC assembly on a wild-type and mutant TATA-containing promoter (Jacob et al. 1994). The authors found a 12 -fold defect in initiation compared to only a 4-fold defect in PIC assembly on the mutant TATA promoter, suggesting a role for the TATA box in a step after PIC assembly.

On the basis of our results, as well as previous studies, we propose that the conformation of the TBP-DNA complex is critical in determining the activity of the PIC. At the HIS4mTATA promoter, TBP cannot interact normally with the $\mathrm{G}-\mathrm{C}$ sequence as the DNA binding sur- 
face of TBP is incompatible with the G-C DNA minor groove (Kim et al. 1993a,b). Although the transcription machinery is stably recruited to the mutant promoter, this lack of normal TBP binding gives rise to a complex in which Pol II is compromised in synthesis of 30 nucleotide transcripts. This may be due in part to a failure of TBP to distort the DNA as is seen on authentic TATA elements (Kim et al. 1993a,b). Thus, the TATA box may play a dual role in the initiation process, first as a target of TBP binding in which it nucleates the assembly of the PIC, and second, in promoting the activity of the PIC through its stereospecific interaction with TBP.

\section{Enhancement of PIC formation by AH and VP16 activators}

In the yeast transcription system, the AH and VP16 activators modestly stimulate initiation in single-round transcription assays (two- to threefold for $\mathrm{AH}$ and threefold for VP16). This modest effect is caused by several factors. First, our templates have a single binding site for the activator for reasons discussed above. Second, our templates were not assembled into chromatin, likely resulting in increased basal transcription. Third, singleround assays show less stimulation by certain activators compared with multiround transcription (Fig. 2b). For Gal4-AH and Gal4-VP16, we observe a two- to threefold stimulation of all PIC components except TBP, which corresponds exactly to the effects observed in singleround transcription. For Gal4-AH, this stimulation seems due to an effect on recruitment of TFIID and TFIIA. First, Gal4-AH is not sufficient to recruit holoenzyme subunits to the promoter in TBP and TFIIA mutant extracts. Second, Gal4-AH stimulation of TFIID and TFIIA binding was unaffected in extracts in which holoenzyme recruitment was defective (data not shown). Finally, in the presence of Gal4-AH, the levels of TFIID and TFIIA at the promoter are not decreased by disruption of holoenzyme recruitment. In contrast, Gal4-VP16 can recruit holoenzyme components and certain $\mathrm{TAF}_{\mathrm{II}} \mathrm{S}$ in a promoter-independent reaction. This promoter-independent recruitment is enhanced by multiple copies of the Gal4-binding site (data not shown). When multiple binding sites are used, $\mathrm{AH}$ can also begin to recruit holoenzyme and certain $\mathrm{TAF}_{\mathrm{II}} \mathrm{S}$, although not as strongly as VP16 (data not shown). These results suggest that the particular activator used, as well as the number of activators at the promoter, can alter the mechanism of factor recruitment to the promoter.

Our results are consistent with a large body of work indicating that activators can stimulate transcription by recruitment of PIC components (Ptashne and Gann 1997). Activators can enhance the rate and extent of TFIID-TFIIA recruitment (Lieberman and Berk 1994; Chi et al. 1995; Kobayashi et al. 1998), and they can enhance assembly of holoenzyme components at the promoter (Choy and Green 1993). Also, artificial recruitment of TBP, $\mathrm{TAF}_{\mathrm{II}} \mathrm{s}$, or holoenzyme components to the promoter promotes high levels of initiation in vivo (Keaveney and Struhl 1998 and references therein).
In summary, the combined genetic and biochemical approach used here should be useful for further probing the mechanism of PIC assembly, elucidating the biochemical defects caused by genetically isolated mutations in transcription factors, and exploring intermediates in the initiation process.

\section{Materials and methods}

Yeast strains

Yeast strains are listed in Table 1.

Preparation of nuclear extracts

Yeast nuclear extracts were prepared from 2-8 liter cultures of the indicated strains grown at the permissive temperature as described in Kang et al. (1995) and on the world wide web at www.fhcrc.org/science/basic/labs/hahn.

\section{Recombinant proteins}

All recombinant proteins were expressed in Escherichia. coli. rTBP was a gift from J. Geiger. rTFIIA was prepared as described in Ranish et al. (1992) after separately expressing the Toal and Toa2 subunits in E. coli. rTFIIB was purified by chromatography on BioRex 70 and Hydroxylapatite resins (Bio-Rad). His-tagged rSrb2 was purified by Nickel-agarose chromatography (Qiagen) under denaturing conditions, and renatured after purification. Gal4-VP16 was purified by polyethyleneimine precipitation followed by chromatography on S-Sepharose (Pharmacia). Gal4$\mathrm{AH}$ was purified by polyethyleneimine precipitation followed by chromatography on DEAE-Sepharose, and S-Sepharose (Pharmacia).

\section{Plasmid templates}

For transcription assays on plasmid templates, pSH515 and pSH514 were used. pSH515 contains 144 bp of the HIS4 core promoter, $(-141$ to +3 with respect to the translation start site) including the TATA box and RNA start sites, cloned downstream of a single Gal4-binding site. In addition, the bases at four positions around the TATA box were changed to eliminate potential cryptic TATA sequences (sequence available upon request). pSH514 is identical to pSH515 except that the TATA box (TATATAATA) was replaced with the sequence TACCCGGGA. pSH388 contains 144 bp of the HIS4 core promoter, $(-141$ to +3 with respect to the translation start site) including the TATA box and RNA start sites, cloned downstream of three Gal4-binding sites. pSH388 lacks the sequence recognized by the primer used for primer extension in all of the experiments.

\section{Biotinylated templates}

Biotinylated templates were prepared by PCR with either pSH515 or pSH514 as templates, biotinylated, upstream primer p965 (5'-biotin-TAATGCAGCTGGCACGACAGG-3') and various downstream primers. p965 is located $\sim 280$ bp upstream from the Gal4 site. For synthesis of the HIS4 and HIS4mTATA templates, downstream primer pNot (5'-GGCCGCTCTAGCTGCATTAATG-3') was used with both pSH515 and pSH514 to produce 594-bp products. For synthesis of the Promoterless template, p965 was used with primer BKS7 (5'-TACCGAGCTCGAATTCGGAGG-3') and pSH515, which resulted in production of a 306-bp fragment that ends 14-bp downsteram of the 
Table 1. Strain list

\begin{tabular}{|c|c|c|}
\hline Strain & Genotype & Reference \\
\hline BWG $1-7 \mathrm{a}$ & MATa leu2-3,112 his4-519 ade1-100 ura3-52 & Pfeifer et al. (1987) \\
\hline SHY109 & $\begin{array}{l}\text { MAT } 1 \text { leu2-3,112 ura3-52 his4-519 } \Delta \text { toa1::HIS4/pSH1-25 (ARS CEN LEU2 } \\
\text { toa1-25) }\end{array}$ & Kang et al. (1995) \\
\hline SHY114 & $\begin{array}{l}\text { MAT leu2-3,112 ura3-52 his4-519 } \Delta \text { toa2:: HIS4/pSH2-3 (ARS CEN LEU2 } \\
\text { toa2-3) }\end{array}$ & Kang et al. (1995) \\
\hline SHY70 & $\begin{array}{l}\text { MAT } \alpha \text { ade6 leu2-3,112 his4-519 ura3-52 } \Delta \text { spt15::HIS4/pSH254 (ARS CEN } \\
\text { LEU2 spt15 (TBPI143N)) }\end{array}$ & Reddy and Hahn (1991) \\
\hline Z561 & MATa his34200 leu2-3,112 lys2-801 ura3-52 & Thompson and Young (1995) \\
\hline Z525 & 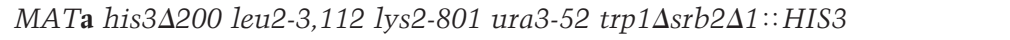 & Gadbois et al. (1997 and pers. comm.) \\
\hline Z562 & 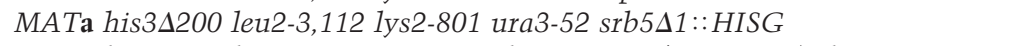 & Gadbois et al. (1997 and pers. comm.) \\
\hline Z628 & 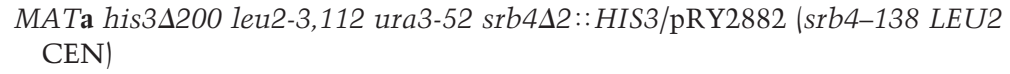 & Gadbois et al. (1997 and pers. comm.) \\
\hline Z649 & MATa his3د200 leu2-3,112 ura3-52 & Gadbois et al. (1997 and pers. comm.) \\
\hline SHY243 & $\begin{array}{l}\text { MATaAde }{ }^{-} \text {leu2-3,112 ura3-52 his4-519 sua74::HIS4/pRK68.42 (ARS CEN } \\
\text { sua7E62G) }\end{array}$ & $\begin{array}{l}\text { this work; Knaus et al. (1996 and pers. } \\
\text { comm.) }\end{array}$ \\
\hline SHY244 & same as SHY243 but with plasmid pRK68.15 (ARS CEN sua7C149R) & $\begin{array}{l}\text { this work; Knaus et al. (1996 and pers. } \\
\text { comm.) }\end{array}$ \\
\hline SHY245 & same as SHY243 but with plasmid pRK68.30 (ARS CEN sua7G41E) & $\begin{array}{l}\text { this work; Knaus et al. (1996 and pers. } \\
\text { comm.) }\end{array}$ \\
\hline $\mathrm{CTD} \Delta 9$ & 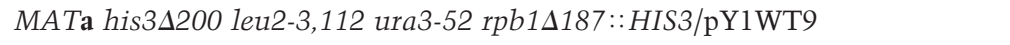 & West and Corden (1995) \\
\hline
\end{tabular}

CTD9 contains the RPB1 gene on an ARS CEN plasmid with RPB1 mutated so that it has nine copies of the CTD repeat.

Gal4 site. PCR products were extracted with phenol:chloroform (1:1), ethanol precipitated, and purified with S300 columns (Pharmacia). DNAs were quantitated by a fluorescent dye binding assay (PanVera Corp.)

\section{Immobilized templates}

Dynabeads M-280 Streptavidin (Dynal) were concentrated with a magnetic particle concentrator (MPC) (Dynal) and washed twice in Buffer $\mathrm{T}$ [10 mM Tris (pH 7.5), 1 mM EDTA, 1 M sodium chloride] $(\sim 1 \mathrm{ml}$ Buffer $\mathrm{T} / \mathrm{mg}$ beads). The beads were resuspended in Buffer T at $10 \mathrm{mg} / \mathrm{ml}$. NP-40 was added to $0.003 \%$ to prevent beads from sticking to the sides of tube. Dynabeads were incubated with $\sim 20$ fmole biotinylated template $/ \mu \mathrm{g}$ bead in Buffer $\mathrm{T}$ for $30 \mathrm{~min}$ at room temperature with constant agitation. The immobilized templates were concentrated with the MPC, and washed in Buffer $\mathrm{T}(\sim 1 \mathrm{ml}$ Buffer $\mathrm{T} / \mathrm{mg}$ beads). Immobilized templates were blocked in block buffer $(1 \mathrm{ml} / \mathrm{mg}$ beads), for $15 \mathrm{~min}$ at room temperature. Block buffer consists of transcription buffer [10 mM HEPES (pH 7.6), $100 \mathrm{~mm}$ potassium glutamate, $10 \mathrm{~mm}$ magnesium acetate, $5 \mathrm{~mm}$ EGTA, $3.5 \%$ glycerol], containing $60 \mathrm{mg} / \mathrm{ml}$ casein (Sigma, C-5890), $5 \mathrm{mg} / \mathrm{ml}$ polyvinylpyrrolidone (USB, 20611), and $2.5 \mathrm{~mm}$ DTT. The beads were concentrated with the MPC, washed three times in transcription buffer, and resuspended in transcription buffer at 10 $\mathrm{mg} / \mathrm{ml}$. Immobilized templates were prepared fresh before each experiment.

\section{In vitro transcription with plasmid templates}

Transcription reactions with plasmid templates were performed as described in Ranish and Hahn (1991) and on the world wide web at www.fhcrc.org/science/basic/labs/hahn/. Reaction components were preincubated for 35-40 min at room temperature, after which $1 \mu \mathrm{l}$ of $10 \mathrm{~mm}$ rNTPs ( $2.5 \mathrm{~mm}$ each) was added to the reactions. For single-round measurements, two methods were used. In the first method, sarkosyl was added to $0.2 \%, 45-60 \mathrm{sec}$ after addition of rNTPs. Reactions were stopped 2-10 min later by the addition of $200 \mu$ of stop solution (0.1 M sodium acetate, $10 \mathrm{~mm}$ EDTA, 0.5\% SDS, $10 \mu \mathrm{g} / \mathrm{ml}$ tRNA). A time course showed that RNA synthesis was complete after $2 \mathrm{~min}$. In the second method, stop solution was added 2 min after addition of rNTPs. Both methods gave comparable results. For multiround measurements, reactions were stopped 40-60 min after rNTP addition. Samples were phenol/chloroform extracted, and precipitated with ethanol. Products were analyzed by primer extension as described in Ranish and Hahn (1991), or by S1 nuclease protection as decribed in Cormack and Struhl (1992). S1 analysis was performed with a 5'-end-labeled oligonucleotide (5'-GTAAACTATTGTATTACTATTACACAGCGCAGGGTGTAG) with a short mismatch to the template strand at the 3' end. Quantitation was performed with a PhosphorImager (Molecular Dynamics).

In vitro transcription with immobilized templates

Reactions were scaled up twofold from the standard transcription reaction to $50 \mu 1$. On ice, $120-180 \mu \mathrm{g}$ of nuclear extract was mixed with $20 \mu \mathrm{l}$ of $2.5 \times$ transcription mix, $0.05 \%$ NP- 40 , and 20-24 $\mu \mathrm{l}$ of transcription buffer to bring the final volume to 44 $\mu 1$. After a $10 \mathrm{~min}$ incubation at room temperature, the mix was spun at $9 \mathrm{~K} \mathrm{rpm}$ for $2 \mathrm{~min}$ in an Eppendorf microfuge at $4^{\circ} \mathrm{C}$. This step removed insoluble material that bound to the magnetic beads. The supernatant was transferred to a new tube on ice, and recombinant factors were added (where indicated). After addition of $\sim 250 \mathrm{ng}$ HaeIII-digested E. coli DNA to reactions, $2.5 \mu \mathrm{l}$ of immobilized template is added. For activated reactions, $2.5 \mu \mathrm{l}$ of template is incubated with $60 \mathrm{ng} \mathrm{Gal} 4-\mathrm{AH}$ for $\sim 10 \mathrm{~min}$ at room temperature before addition to the reaction. After a 40 min incubation, immobilized templates were concentrated with the MPC, and washed three times with $150 \mu \mathrm{l}$ of transcription buffer containing $0.05 \%$ NP-40, $0.5 \mathrm{mg} / \mathrm{ml} \mathrm{BSA}$, and 2.5 mM DTT. After resuspension in $50 \mu \mathrm{l}$ of transcription buffer containing $12 \mathrm{~mm}$ phosphocreatine, 400 ng creatine phosphokinase, $2.5 \mathrm{~mm} \mathrm{DTT}, 0.5 \mathrm{mg} / \mathrm{ml} \mathrm{BSA}$, and 20 units of ribonuclease inhibitor, transcription was initiated by addition of $100 \mu \mathrm{M}$ of 
each rNTP. Reactions were stopped after 2-10 min by addition of $400 \mu \mathrm{l}$ of stop solution. The supernatant was removed from the beads, phenol/chloroform extracted, and ethanol precipitated. Products were mapped by primer extension as described above, except that actinomycin $\mathrm{C}_{1}$ was included $(15 \mu \mathrm{g} / \mathrm{ml})$ during the extension reaction. In Figure 8, primer extension products were resolved on a 6\% Quickpoint sequencing gel according to the manufacturer's protocol (NOVEX).

\section{Analysis of proteins on immobilized templates}

To analyze proteins bound to immobilized templates, $100 \mu \mathrm{l}$ of immobilized transcription reactions were used. After preincubation of extract with immobilized templates, the templates were concentrated with the MPC, and washed three times with $300 \mu \mathrm{l}$ of transcription buffer containing $0.05 \%$ NP-40, $0.5 \mathrm{mg} /$ $\mathrm{ml} \mathrm{BSA}$, and $2.5 \mathrm{~mm}$ DTT. Templates were resuspended in 12.5 ul of Buffer \#3 (NEBiolabs) with 60 units of PstI. After incubation for $30 \mathrm{~min}$ at $37^{\circ} \mathrm{C}$ with constant agitation, the beads were concentrated with the MPC, and the supernatant was removed. A total of $12.5 \mu \mathrm{l}$ of $2 \times$ SDS loading buffer was added. Samples were boiled for $3 \mathrm{~min}$, and resolved on $12 \%$ SDS-polyacrylamide gels. In Figure 8, samples were resolved on NuPAGE $4 \%-12 \%$ polyacrylamide gels according to the manufacturer's protocol (NOVEX). Proteins were electroblotted to Immobilon membranes (Millipore), and detected by use of Amersham or Pierce ECL kits. Quantitation was performed by densitometry with IQMACv1.2 software (Molecular Dynamics). When possible, the relative levels of subunits were determined by standard curves that were generated with increasing amounts of nuclear extract. In cases in which quantitation was based on absolute densitometry values, accuracy is limited by the lack of linearity in the dose response of the detection system and the antibody-antigen interaction. However, in most cases the results were reproduced at least three times.

\section{Acknowledgments}

We thank members of the Hahn laboratory, past and present, for their thoughtful insights, and A. Krumm, R. Reeder, and T. Tsukiyama for comments on the manuscript. A. Berk suggested important controls. We thank E. Gadbois, R. Young, R. Knauss, L. Guarente, M. Carey, and J. Corden for strains. We also thank E. Gadbois, R. Young, J. Reese, M. Green, C.-Y. Ho, M. Swanson, Y. Nakatani, N. Thompson, D. Burgess, S. Buratowski, and C. Denis for antibodies. R. Kornberg and Y.-J. Kim generously provided E. coli strains expressing recombinant TFIIE and Med6 subunits used for antibody production. We thank K. Coachman and G. Schimmack for antibody production, J. Geiger for rTBP, S. Hurst for rTFIIB, and S. Miller for rSrb2.

This work was funded by a National Science Foundation predoctoral fellowship (J.A.R.), National Cancer Institute grant T32CA09657 (N.Y.), and National Institutes of Health grant GM42551 and a Leukemia Society Scholar Award to S.H. S.H. is an associate investigator of the Howard Hughes Medical Institute.

\section{References}

Auble, D.T. and S. Hahn. 1993. An ATP-dependent inhibitor of TBP binding to DNA. Genes \& Dev. 7: 844-856.

Bryant, G.O., L.S. Martel, S.K. Burley, and A.J. Berk. 1996. Radical mutations reveal TATA-box binding protein surfaces required for activated transcription in vivo. Genes \& Dev. 10: $2491-2504$.
Bushnell, D.A., C. Bamdad, and R.D. Kornberg. 1996. A minimal set of RNA polymerase II transcription protein interactions. J. Biol. Chem. 271: 20170-20174.

Chang, M. and J.A. Jaehning. 1997. A multiplicity of mediators: Alternative forms of transcription complexes communicate with transcriptional regulators. Nucleic Acids Res. 25: 4861-4865.

Chi, T. and M. Carey. 1996. Assembly of the isomerized TFIIA-TFIID-TATA ternary complex is necessary and sufficient for gene activation. Genes \& Dev. 10: 2540-2550.

Chi, T., P. Lieberman, K. Ellwood, and M. Carey. 1995. A general mechanism for transcriptional synergy by eukaryotic activators. Nature 377: 254-257.

Choy, B. and M.R. Green. 1993. Eukaryotic activators function during multiple steps of preinitiation complex assembly. Nature 366: 531-536.

Cormack, B.P. and K. Struhl. 1992. The TATA-binding protein is required for transcription by all three nuclear RNA polymerases in yeast cells. Cell 69: 685-696.

DeJong, J., R. Bernstein, and R.G. Roeder. 1995. Human general transcription factor TFIIA: Characterization of a cDNA encoding the small subunit and requirement for basal and activated transcription. Proc. Natl. Acad. Sci. 92: 3313-3317.

Gadbois, E.L., D.M. Chao, J.C. Reese, M.R. Green, and R.A. Young. 1997. Functional antagonism between RNA polymerase II holoenzyme and global negative regulator NC2 in vivo. Proc. Natl. Acad. Sci. 94: 3145-3150.

Geiger, J.H., S. Hahn, S. Lee, and P.B. Sigler. 1996. Crystal structure of the yeast TFIIA/TBP/DNA complex. Science 272: 830-836.

Gerber, H.-P., M. Hagmann, K. Seipel, O. Gerogiev, M.A.L. West, Y. Litingtung, W. Schaffner, and J.L. Corden. 1995. RNA polymerase II C-terminal domain required for enhancer-driven transcription. Nature 374: 660-662.

Grant, P.A., D. Schieltz, M.G. Pray-Grant, D.J. Steger, J.C. Reese, J.R. Yates, 3rd, and J.L. Workman. 1998. A subset of TAF(II)s are integral components of the SAGA complex required for nucleosome acetylation and transcriptional. Cell 94: 45-53.

Hampsey, M. 1998. Molecular genetics of the RNA polymerase II general transcriptional machinery. Microbiol. Mol. Biol. Rev. 62: 465-503.

Hengartner, C.J., C.M. Thompson, J. Zhang, D.M. Chao, S.-M. Liao, A.J.

Koleske, S. Okamura, and R.A. Young. 1995. Association of an activator with an RNA polymerase II holoenzyme. Genes \& Dev. 9: 897-910.

Hoopes, B.C., J.F. LeBlanc, and D.K. Hawley. 1998. Contributions of the TATA box sequence to rate-limiting steps in transcription initiation by RNA polymerase II. J. Mol. Biol. 277: 1015-1031.

Jacob, G.A., J.A. Kitzmiller, and D.S. Luse. 1994. RNA polymerase II promoter strength in vitro may be reduced by defects at initiation or promoter clearance. J. Biol. Chem. 269: 36553663.

Kang, J.J., D.T. Auble, J.A. Ranish, and S. Hahn. 1995. Analysis of the yeast transcription factor TFIIA: Distinct functional regions and a polymerase II-specific role in basal and activated transcription. Mol. Cell. Biol. 15: 1234-1243.

Kassavetis, G.A., A. Kumar, G.A. Letts, and E.P. Geiduschek. 1998. A post-recruitment function for the RNA polymerase III transcription-initiation factor IIIB. Proc. Natl. Acad. Sci. 95: 9196-9201.

Keaveney, M. and K. Struhl. 1998. Activator-mediated recruitment of the RNA polymerase II machinery is the predominant mechanism for transcriptional activation in yeast. Mol. 
Cell 1: 917-924.

Kim, J.L., D.B. Nikolov, and S.K. Burley. 1993a. Co-crystal structure of TBP recognizing the minor groove of a TATA element. Nature 365: 520-527.

Kim, Y., J.H. Geiger, S. Hahn, and P.B. Sigler. 1993b. Crystal structure of a yeast TBP/TATA-box complex. Nature 365: 512-520.

Knaus, R., R. Pollock, and L. Guarente. 1996. Yeast SUB1 is a suppressor of TFIIB mutations and has homology to the human co-activator PC4. EMBO J. 15: 1933-1940.

Kobayashi, N., P.J. Horn, S.M. Sullivan, S.J. Triezenberg, T.G. Boyer, and A.J. Berk. 1998. DA-complex assembly activity required for VP16C transcriptional activation. Mol. Cell Biol. 18: 4023-4031.

Kokubo, T., M.J. Swanson, J.I. Nishikawa, A.G. Hinnebusch, and Y. Nakatani. 1998. The yeast TAF145 inhibitory domain and TFIIA competitively bind to TATA-binding protein. Mol. Cell Biol. 18: 1003-1012.

Koleske, A.J. and R.A. Young. 1995. The RNA polymerase II holoenzyme and its implications for gene regulation. Trends Biochem. Sci. 20: 113-116.

Lee, D. and J.T. Lis. 1998. Transcriptional activation independent of TFIIH kinase and the RNA polymerase II mediator in vivo. Nature 393: 389-392.

Lee, T.I. and R.A. Young. 1998. Regulation of gene expression by TBP-associated proteins. Genes \& Dev. 12: 1398-1408.

Lee, T.I., J.J. Wyrick, S.S. Koh, E.G. Jennings, E.L. Gadbois, and R.A. Young. 1998. Interplay of positive and negative regulators in transcription initiation by RNA polymerase II holoenzyme. Mol. Cell Biol. 18: 4455-4462.

Liao, S.M., I.C. Taylor, R.E. Kingston, and R.A. Young. 1991. RNA polymerase II carboxy-terminal domain contributes to the response to multiple acidic activators in vitro. Genes \& Dev. 5: 2431-2440.

Lieberman, P.M. and A.J. Berk. 1994. A mechanism for TAFs in transcriptional activation: Activation domain enhancement of TFIID-TFIIA-promoter DNA complex formation. Genes \& Dev. 8: 995-1006.

McNeil, J.B., H. Agah, and D. Bentley. 1998. Activated transcription independent of the RNA polymerase II holoenzyme in budding yeast. Genes \& Dev. 12: 2510-2521.

Myer, V.E. and R.A. Young. 1998. RNA polymerase II holoenzymes and subcomplexes. J. Biol. Chem. 273: 27757-27760.

Myers, L.C.,C.M. Gustafsson, D.A. Bushnell, M. Lui, H. Erdjument-Bromage, P. Tempst, and R.D. Kornberg. 1998. The Med proteins of yeast and their function through the RNA polymerase II carboxy-terminal domain. Genes \& Dev. 12: $45-54$.

Orphanides, G., T. Lagrange, and D. Reinberg. 1996. General initiation factors of RNA Polymerase II. Genes \& Dev. 10: $2657-2683$.

Ozer, J., K. Mitsouras, D. Zerby, M. Carey, and P.M. Lieberman. 1998. Transcription factor IIA derepresses TATA-binding protein (TBP)-associated factor inhibition of TBP-DNA binding. J. Biol. Chem. 273: 14293-14300.

Pardee, T.S., C.S. Bangur, and A.S. Ponticelli. 1998. The N-terminal region of yeast TFIIB contains two adjacent functional domains involved in stable RNA polymerase II binding and transcription start site selection. J. Biol. Chem. 273: 1785917864.

Pfeifer, K., T. Prezant, and L. Guarente. 1987. Yeast HAP1 activator binds to two upstream activation sites of different sequence. Cell 49: 19-27.

Pinto, I., W.-H. Wu, J.G. Na, and M. Hampsey. 1994. Characterization of sua7 mutations defines a domain of TFIIB in- volved in transcription start site selection in yeast. J. Biol. Chem. 269: 30569-30573.

Ptashne, M. and A. Gann. 1997. Transcriptional activation by recruitment. Nature 386: 569-577.

Ranish, J.A. and S. Hahn. 1991. The yeast general transcription factor TFIIA is composed of two polypeptide subunits. $J$. Biol. Chem. 266: 19320-19327.

Ranish, J.A., W.S. Lane, and S. Hahn. 1992. Isolation of two genes that encode subunits of the yeast transcription factor IIA. Science 255: 1127-1129.

Reese, J.C., L. Apone, S.S. Walker, L.A. Griffin, and M.R. Green. 1994. Yeast TAFIIS in a multisubunit complex required for activated transcription. Nature 371: 523-527.

Sandaltzopoulos, R. and P.B. Becker. 1998. Heat shock factor increases the reinitiation rate from potentiated chromatin templates. Mol. Cell. Biol. 18: 361-367.

Stargell, L.A. and K. Struhl. 1995. The TBP-TFIIA interaction in the response to acidic activators in vivo. Science 269: 75-78.

Struhl, K. 1996. Chromatin structure and RNA polymerase II connection: Implications for transcription. Cell 84: 179-182.

Tan, S., Y. Hunziker, D.F. Sargent, and T.J. Richmond. 1996. Crystal structure of a yeast TFIIA/TBP/DNA complex. $\mathrm{Na}$ ture 381: 127-151.

Thompson, C.M. and R.A. Young. 1995. General requirement for RNA polymerase II holoenzymes in vivo. Proc. Nat1. Acad. Sci. 92: 4587-4590.

Weideman, C.A., R.C. Netter, L.R. Benjamin, J.J. McAllister, L.A. Schmiedekamp, R.A. Coleman, and B.F. Pugh. 1997. Dynamic interplay of TFIIA, TBP and TATA DNA. J. Mol. Biol. 271: 61-75.

West, M.L. and J.L. Corden. 1995. Construction and analysis of yeast RNA polymerase II CTD deletion and substitution mutations. Genetics 140: 1223-1233.

Wilson, C.J., D.M. Chao, A.N. Imbalzano, G.R. Schnitzler, R.E. Kingston, and R.A. Young. 1996. RNA polymerase II holoenzyme contains SWI/SNF regulators involved in chromatin remodeling. Cell 84: 235-244.

Wobbe, C.R. and K. Struhl. 1990. Yeast and human TATA-binding proteins have nearly identical DNA sequence requirements for transcription in vitro. Mol. Cell Biol. 10: 38593867.

Yean, D. and J. Gralla. 1997. Transcription reinitiation rate: A special role for the TATA box. Mol. Cell. Biol. 17: 38093816.

Zawel, L., K.P. Kuman, and D. Reinberg. 1995. Recycling of the general transcription factors during RNA polymerase II transcription. Genes \& Dev. 9: 1479-1490. 


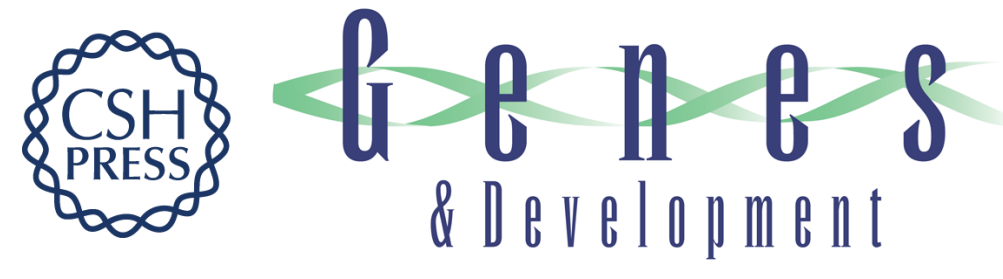

\section{Intermediates in formation and activity of the RNA polymerase II preinitiation complex: holoenzyme recruitment and a postrecruitment role for the TATA box and TFIIB}

Jeffrey A. Ranish, Natalya Yudkovsky and Steven Hahn

Genes Dev. 1999, 13:

Access the most recent version at doi:10.1101/gad.13.1.49

References This article cites 54 articles, 33 of which can be accessed free at:

http://genesdev.cshlp.org/content/13/1/49.full.html\#ref-list-1

License

Email Alerting Receive free email alerts when new articles cite this article - sign up in the box at the top Service right corner of the article or click here.

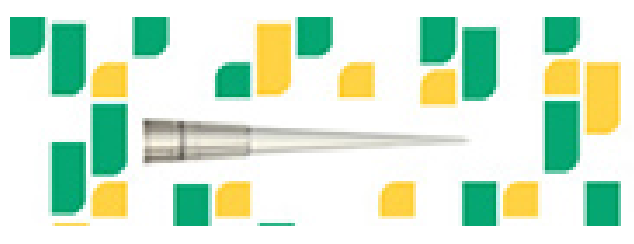

Focused on your science. 